\title{
Biologically unavoidable sequences
}

\author{
Samuel A. Alexander \\ Department of Mathematics \\ The Ohio State University \\ Ohio, U.S.A. \\ alexander@math.ohio-state.edu
}

Submitted: Jan 7, 2013; Accepted: Jan 28, 2013; Published: Feb 5, 2013

Mathematics Subject Classifications: 05C63, 92B10

\begin{abstract}
A biologically unavoidable sequence is an infinite gender sequence which occurs in every gendered, infinite genealogical network satisfying certain tame conditions. We show that every eventually periodic sequence is biologically unavoidable (this generalizes König's Lemma), and we exhibit some biologically avoidable sequences. Finally we give an application of unavoidable sequences to cellular automata.
\end{abstract}

\section{Introduction}

The following definition is motivated by biological considerations. The idea of modelling the biosphere with a directed graph goes at least back to Hennig [10], more recently (and more formally) to Dress, Moulton, Steel and Wu [6]. The idea of including vertices for future organisms was made explicit in Kornet, Metz, and Schellinx [12]. The (simplifying) assumption of infinitely many vertices was made explicit in Alexander [2]. We hope to submit the results of this paper as an answer to Sturmfels [16].

Definition 1. Let $n>0$. An infinite $n$-gendered population is a directed graph $G=$ $(V, E)$, together with a map $v \mapsto t(v) \in \mathbb{R}$ assigning birthdates to vertices and a map $e \mapsto g(e) \in\{1, \ldots, n\}$ assigning genders to edges, satisfying the following conditions (we call $v$ a parent of $u$ if $(v, u) \in E$, we define child similarly, and we define ancestor and descendant in the obvious (strict) way):

- (A1) There are only finitely many parentless vertices (call them roots).

- (A2) Each vertex has only finitely many children.

- (A3) For every $r \in \mathbb{R},\{v \in V: t(v) \leqslant r\}$ is finite, and for each $(u, v) \in E$, $t(u)<t(v)$. 
- (A4) $|V|=\infty$.

- $(n$-Gendered) For every non-root $u$ and every $1 \leqslant i \leqslant n, u$ has a parent $v$ with $g(v, u)=i$.

Note that A3 implies $G$ is a DAG and is reverse-well-founded, i.e., has no infinite reverse-directed path. Thus every non-root is a descendant of some root. Although Definition 1 stipulates that edges be gendered, we will often consider special cases where vertices are gendered, implicitly giving each edge the gender of its initial vertex.

Definition 2. Let $n>0$. An infinite sequence $s=\left(s_{1}, s_{2}, \ldots\right) \in\{1, \ldots, n\}^{\mathbb{N}}$ is biologically unavoidable if every infinite $n$-gendered population realizes $s$ - by which we mean there is a vertex sequence $v_{1}, v_{2}, \ldots$ with each $v_{i}$ a parent of $v_{i+1}$ and each $g\left(v_{i}, v_{i+1}\right)=s_{i}$. If $s$ is not biologically unavoidable, it is biologically avoidable.

A priori, biological unavoidability appears ill-defined. The following lemma shows that it is well-defined.

Lemma 3. Suppose $s \in\{1, \ldots, n\}^{\mathbb{N}}$ and at the same time $s \in\{1, \ldots, m\}^{\mathbb{N}}$. Then $s$ is realized in every infinite $n$-gendered population if and only if $s$ is realized in every infinite m-gendered population.

Proof. Without loss of generality, $n<m$. If there is an infinite $m$-gendered population which fails to realize $s$, delete all edges with genders $>n$ to obtain an infinite $n$-gendered population which fails to realize $s$. Conversely, suppose there is an $n$-gendered population $P$ failing to realize $s$. Inductively it suffices to show there's an $(n+1)$-gendered population failing to realize $s$. Let $P^{\prime}$ be a disjoint copy of $P$. To the graph $P^{\prime} \sqcup P$, add two $(n+1)$ gendered edges, $\left(v^{\prime}, u\right)$ and $\left(v, u^{\prime}\right)$, for every $n$-gendered edge $(v, u)$ in $P$. It's easy to see this makes $P \sqcup P^{\prime}$ an $(n+1)$-gendered population which fails to realize $s$.

In Section 2, we will show that every eventually periodic sequence is biologically unavoidable.

In Section 3, we will establish the existence of biologically avoidable sequences. This nontrivial fact involves a couple of unexpected enumerative combinatorial arguments.

In Section 4, we will show that there are biologically avoidable sequences from $\{1,2\}^{\mathbb{N}}$ which contain no gender repeated thrice in a row.

In Section 5, we will give an unexpected application to cellular automata: an alternate proof of a result about spaceship speed limits in Conway's Life-like Games, first proved by Nathaniel Johnston [11].

\section{Eventually periodic implies biologically unavoidable}

Before proving the unavoidability of eventually periodic sequences, a small amount of machinery must be developed. For the remainder of this section, let $P=(V, E)$ be an infinite $n$-gendered population, $n>0$. 
Definition 4. For $i \in \mathbb{N}$, we define the set $V_{i} \subseteq V$ as follows: a vertex $u \in V$ lies in $V_{i}$ if and only if there is some root $r$ and some directed path from $r$ to $u$ of length $\leqslant i$.

Thus $V_{0}$ is the set of roots, $V_{1}$ contains the roots and their children, and so on.

Lemma 5. For every $i \in \mathbb{N}, V_{i}$ is finite.

Proof. Follows trivially from A1 and A2.

Lemma 6. For every $1 \leqslant i \leqslant n$, there is a map $i^{*}: V \backslash V_{0} \rightarrow V$ such that for every $u \in V \backslash V_{0}$ we have $\left(i^{*} u, u\right) \in E$ and $g\left(i^{*} u, u\right)=i$.

Proof. By the axiom of choice and the $n$ Gendered assumption on $P$.

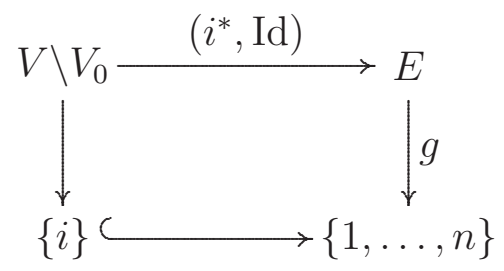

In case $n=2$, one might refer to $1^{*}$ and $2^{*}$ as motherhood and fatherhood maps, in some order. We will write $i^{*} j^{*}$ for $i^{*} \circ j^{*}$.

Definition 7. If $s=\left(s_{1}, s_{2}, \ldots\right)$ is a periodic sequence on $\{1, \ldots, n\}$, with period $p$, and if $u \in V$, then we define the $s$-path to $u$, a finite directed path, written $s^{*} u$, as follows.

1. If $u \in V_{p-1}$ then $s^{*} u=(u)$ (the length 0 path).

2. Otherwise,

$$
s^{*} u=\left(s_{1}^{*} \cdots s_{p q}^{*} u, s_{2}^{*} \cdots s_{p q}^{*} u, \ldots, s_{p q-1}^{*} s_{p q}^{*} u, s_{p q}^{*} u, u\right),
$$

where $q$ is maximal such that $s_{1}^{*} \cdots s_{p q}^{*} u$ is defined. (Some such $q>0$ exists lest $u$ lie in $V_{p-1}$, and such $q$ are bounded above due to Assumption A3.)

For example, suppose $n=2$ and $\{1,2\}=\{M, F\}$. If $s=(M, M, M, \ldots)$, then $s^{*} u$ is obtained as follows: start with $u$, go to $u$ 's father, go to his father, and so on until a root is reached; then reverse the order of the resulting path. If $s=(M, F, F, M, F, F, \ldots)$, then $s^{*} u$ is obtained by starting at $u$, taking his mother, taking her mother, taking her father, and repeating this three-step process until too close to a root to continue; and then reversing the resulting path.

Lemma 8. Let $s, p$ be as in Definition 7 . For any $u \in V, s^{*} u$ is a finite directed path starting at a vertex in $V_{p-1}$ and ending at $u$, and if $s^{*} u=\left(v_{1}, \ldots, v_{r}\right)$ then for all $1 \leqslant i<r$, $g\left(v_{i}, v_{i+1}\right)=s_{i}$.

Proof. To see that $s^{*} u$ starts in $V_{p-1}$, write $s^{*} u=\left(v_{1}, \ldots, v_{r}\right)$ and assume $v_{1} \notin V_{p-1}$. Then $s_{1}^{*} \cdots s_{p}^{*} v_{1}$ is defined. Let $q$ be as in the definition of $s^{*} u$. By periodicity of $s$,

$$
\begin{aligned}
s_{1}^{*} \cdots s_{p}^{*} v_{1} & =s_{1}^{*} \cdots s_{p}^{*}\left(s_{1}^{*} \cdots s_{p q}^{*} u\right) \\
& =s_{1}^{*} \cdots s_{p}^{*} s_{1}^{*} \cdots s_{p q}^{*} u \\
& =s_{1}^{*} \cdots s_{p(q+1)}^{*} u,
\end{aligned}
$$

violating the maximality of $q$. The rest of the lemma is clear. 
Proposition 9. Every periodic sequence is biologically unavoidable. In fact, if $s$ is a sequence with period $p$, it is realized by a path in $P$ which begins at a vertex in $V_{p-1}$.

Proof. Consider the set (call it $X$ ) of finite directed paths $\left\{s^{*} u\right\}_{u \in V}$ (note $|X|=\infty$ by Assumption A4). Each begins (by Lemma 8) in $V_{p-1}$, and $V_{p-1}$ is finite by Lemma 5 . Thus, there is some $u_{1} \in V_{p-1}$ such that infinitely many members (call them $X_{1}$ ) of $X$ begin at $u_{1}$.

Inductively, suppose I've defined a finite directed path $u_{1}, \ldots, u_{k}$ and an infinite set $X_{k} \subseteq X$ such that

1. For all $\Pi \in X_{k}$, the $k$ th vertex in $\Pi$ is $u_{k}$; and

2. $g\left(u_{i}, u_{i+1}\right)=s_{i}$ for all $0<i<k$.

It follows from Assumptions A1 and A2 that $X_{k}$ contains only finitely many paths of length $\leqslant k$, we may assume it contains no paths so short.

Since each path in $X_{k}$ has $u_{k}$ as $k$ th vertex, each path in $X_{k}$ has some child of $u_{k}$ as $(k+1)$ th vertex. By Assumption A2, $u_{k}$ only has finitely many children. Thus there is a child $u_{k+1}$ of $u_{k}$ such that an infinite subset $X_{k+1}$ of paths in $X_{k}$ have $u_{k+1}$ as $(k+1)$ th vertex.

In particular, $X_{k+1}$ has at least one path, $s^{*} v$ for some $v \in V$. By Lemma 8, $g\left(u_{k}, u_{k+1}\right)=s_{k}$.

This inductively defines $u_{1}, u_{2}, \ldots$ with all the desired properties.

It is also possible to prove Proposition 9 using the compactness theorem from firstorder logic. Compare the proof (see Simpson's book [15]) that weak König's Lemma is equivalent (over $\mathrm{RCA}_{0}$ ) to the compactness theorem.

Theorem 10. Every eventually periodic sequence is biologically unavoidable.

Proof. If $s$ is eventually periodic, write it as $s=t \frown t^{\prime}$ where $t$ is finite of length $k$ and $t^{\prime}$ is periodic. Let $P^{\prime}$ be the population obtained from $P$ by discarding all vertices in $V_{k-1}$; it's easy to see $P^{\prime}$ remains an infinite gendered population. By Proposition 9, there is a directed path in $P^{\prime}$ realizing $t^{\prime}$. This defines a path $u_{1}, u_{2}, \ldots$ in $P$, realizing $t^{\prime}$, and avoiding $V_{k-1}$. Back-extend this path to

$$
\left(t_{1}^{*} \cdots t_{k}^{*} u_{1}, t_{2}^{*} \cdots t_{k}^{*} u_{1}, \ldots, t_{k}^{*} u_{1}, u_{1}, u_{2}, \ldots\right),
$$

which realizes $s$ as desired; this is possible because if not, that would imply $u_{1} \in V_{k-1}$.

This generalizes König's Lemma for trees, which can be seen as the 1-gender case of Theorem 10 with the additional constraint that vertices have only one parent. The following corollary pushes this idea even further.

Corollary 11. There is a subset $V_{0} \subseteq V$, ancestrally closed (whenever $v \in V_{0}$ and $u$ is an ancestor of $v$ then $u \in V_{0}$ ), such that for every eventually periodic sequence $s \in\{1, \ldots, n\}^{\mathbb{N}}, G$ realizes $s$ with a path $p$ entirely in $V_{0}$, with the additional property that every vertex in $V_{0}$ has a descendant on $p$. 
Proof. By Theorem 10 above, combined with Theorem 3 and Proposition 5 from Alexander $[2]$.

Using Theorem 10 we can give a game-theoretical characterization of unavoidable sequences using the notion of guessability discovered by Wadge [17] (pp. 141-145) (and independently by Alexander [1]). Let $s$ be a sequence on $\{1, \ldots, n\}$. In the game $G_{s}, I$ starts by playing an infinite $n$-gendered population $P$. Thereafter, $I I$ plays a path $p$ in $P$ (one vertex per turn) and $I$ tries to guess (making one guess per turn) whether $p$ 's genders have the form $t \frown s$ for some finite $t$. $I$ wins if $I$ 's guesses converge to the correct answer, $I I$ wins otherwise. We leave it an exercise that $I$ has a winning strategy iff $s$ is biologically avoidable. (This holds whether or not II can see I's guesses.)

\section{Existence of Biologically Avoidable Sequences}

One might hope to cleverly generalize the argument from the previous section to nonperiodic gender sequences. In this section we'll show that's impossible. There are sequences which are biologically avoidable. Populations lacking certain gender sequences are analogous to Aronszajn trees (first introduced by Kurepa [13]) in the sense that both provide counterexamples to plausible-seeming generalizations of König's Lemma.

In this and the next section we restrict attention to populations with gendered vertices, implicitly gendering edges according to their initial vertices.

Definition 12. Suppose $P=(V, E)$ is an infinite $n$-gendered population, $s=\left(s_{1}, \ldots, s_{k}\right)$ is a finite sequence, $k>0$, and $V$ is partitioned into heights $H_{1}, H_{2}, \ldots$ We say that $s$ is impossible in $P$ at height $k$ if there is no finite directed path $v_{1}, \ldots, v_{k}$, gendered by $s$, with $v_{1} \in H_{k}$.

Lemma 13. Suppose $s$ is an infinite $\{1, \ldots, n\}$-sequence. If there is an infinite $n$-gendered population $P=(V, E)$ and a partition of $V$ into heights $H_{1}, H_{2}, \ldots$ such that for every $k>0$, some finite restriction of $s$ is impossible in $P$ at height $k$, then $s$ is biologically avoidable.

Proof. If $s$ were realized by $P$, it would be realized by some path, starting with a vertex in some height $H_{k}$, yet $s$ would have some finite restriction impossible at height $k$, impossible. So $s$ is not realized by $P$, so $s$ is biologically avoidable.

We will now define a specific family of infinite 2-gendered populations (generalizing an example suggested by Timothy J. Carlson) designed to take advantage of Lemma 13. Let $\{M, F\}=\{1,2\}$, we will refer to $M$-gendered vertices as males, $F$-gendered vertices as females, and adopt terminology such as son, daughter with the obvious meanings.

Definition 14. Suppose $h: \mathbb{N}^{+} \rightarrow \mathbb{N}^{+}$. The infinite 2-gendered population $T_{h}$ is defined as follows (Figure 1 shows $T_{n \mapsto n}$ ). The vertices of $T_{h}$ are partitioned into successive generations $G_{1}, G_{2}, \ldots$, the $n$th generation consisting of $h(n)$ males $m_{1}^{n}, \ldots, m_{h(n)}^{n}$ and 
$h(n)$ females $f_{1}^{n}, \ldots, f_{h(n)}^{n}$. These vertices are given birthdates, $v \mapsto t(v)$, so that vertices in $G_{i}$ are born before those in $G_{j}$ whenever $i<j$, and within each generation $G_{n}$, $\max \left\{t\left(m_{i}^{n}\right), t\left(f_{i}^{n}\right)\right\}<\min \left\{t\left(m_{j}^{n}\right), t\left(f_{j}^{n}\right)\right\}$ whenever $i<j$. Edges are defined as follows.

- $m_{1}^{1}$ and $f_{1}^{1}$ have no parents.

- $\forall n>0, m_{1}^{n+1}$ has parents $m_{h(n)}^{n}$ and $f_{1}^{n}$.

- $\forall n>0, f_{1}^{n+1}$ has parents $f_{h(n)}^{n}$ and $m_{1}^{n}$.

- $\forall n>0, \forall 0<i<h(n), m_{i+1}^{n}$ has parents $m_{i}^{n}$ and $f_{1}^{n}$.

- $\forall n>0, \forall 0<i<h(n), f_{i+1}^{n}$ has parents $f_{i}^{n}$ and $m_{1}^{n}$.

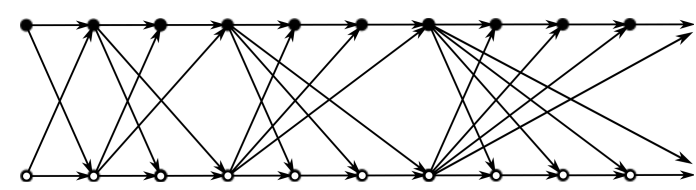

Figure 1: The infinite 2gendered population $T_{n \mapsto n}$. Solid and open vertices correspond to males and females, not necessarily in that order.

Lemma 15. Let $h: \mathbb{N}^{+} \rightarrow \mathbb{N}^{+}$.

1. $T_{h}$ is an infinite 2 -gendered population with roots $m_{1}^{1}$ and $f_{1}^{1}$.

2. If $m_{i}^{n}$ has a daughter, or $f_{i}^{n}$ has a son, then $i=1$.

3. No edge in $T_{h}$ skips an entire generation: if an edge has initial vertex in $G_{i}$, then it has terminal vertex in either $G_{i}$ or $G_{i+1}$.

4. If $B=\left(v_{1}, \ldots, v_{n}\right)$ is a finite directed path of males from $T_{h}$, such that $v_{1} \in G_{i}$ and $v_{n} \in G_{j}$, then for every $i<k<j, B$ contains all the males in $G_{k}$.

Proof. Left to the reader.

Definition 16. When a function $h: \mathbb{N}^{+} \rightarrow \mathbb{N}^{+}$is clear from context, we let $\widehat{m}_{1}, \widehat{m}_{2}, \ldots$ denote the males in $T_{h}$ (over all the generations), ordered ascending by birthdate. Similarly for $\widehat{f}_{1}, \widehat{f}_{2}, \ldots$. We partition $T_{h}$ into heights $H_{1}, H_{2}, \ldots$ by letting each $H_{i}=\left\{\widehat{m}_{i}, \widehat{f}_{i}\right\}$.

The following technical lemma should be compared and contrasted with Cauchy's polygonal number theorem (see Nathanson [14]) which states that every positive integer can be written as a sum of $n n$-gonal numbers, for any $n \geqslant 3$. For example, every natural number has the form $\sum_{p=1}^{b_{1}} p+\sum_{p=1}^{b_{2}} p+\sum_{p=1}^{b_{3}} p$. Also worth comparing is the work of D. Cantor and B. Gordin [4], and more recently of S. Gupta [8].

Lemma 17. Suppose $h: \mathbb{N}^{+} \rightarrow \mathbb{N}^{+}$and $\lim _{n \rightarrow \infty} h(n)=\infty$. For every $u \in \mathbb{N}$, there is some positive integer e which is not of the form

$$
a+\sum_{p=1}^{b} h(c+p)
$$

for any $a, b, c \in \mathbb{N}$ with $c \leqslant u$ and $a \leqslant \max \{h(1), \ldots, h(u)\}$. 
Proof. Let $A=\max \{h(1), \ldots, h(u)\}$. Since $\lim _{n \rightarrow \infty} h(n)=\infty$, there is some $M_{0}$ so big that $h(c+M)>(u+1)(A+1)$ whenever $M \geqslant M_{0}$ and $0 \leqslant c \leqslant u$. Let

$$
M=\max \left\{a+\sum_{p=1}^{b} h(c+p): a \leqslant A, c \leqslant u, b \leqslant M_{0}\right\}
$$

and let $X=\{M+1, M+2, \ldots, M+(u+1)(A+1)+1\}$.

I claim that for every $c \leqslant u, X$ contains at most $A+1$ different numbers of the form $a+\sum_{p=1}^{b} h(c+p)$ with $a \leqslant A$. If not, by the pigeonhole principle there is some particular $a \leqslant A$ and some $b_{1}<b_{2}$ such that $a+\sum_{p=1}^{b_{1}} h(c+p)$ and $a+\sum_{p=1}^{b_{2}} h(c+p)$ are both in $X$, let $d$ be their difference. Then $d=h\left(c+b_{1}+1\right)+\cdots+h\left(c+b_{2}\right) \geqslant h\left(c+b_{2}\right)$. Since $a+\sum_{p=1}^{b_{2}} h(c+p)>M$ (by virtue of being in $X$ ), by definition of $M$ this implies $b_{2} \geqslant M_{0}$, whereby $d \geqslant h\left(c+b_{2}\right)>(u+1)(A+1)$. This is absurd: $X$ is made up of $(u+1)(A+1)+1$ consecutive points, no two of them can have a difference $>(u+1)(A+1)$. The claim is proved.

Given the above claim, the number of numbers in $X$ with the form $a+\sum_{p=1}^{b} h(c+p)$, $a \leqslant A, c \leqslant u$, is at most $(u+1)(A+1): u+1$ choices for $c$, times $A+1$ numbers of the given form for each $c$. Since $|X|>(u+1)(A+1), X$ contains an $e$ as desired.

Proposition 18. If $h: \mathbb{N}^{+} \rightarrow \mathbb{N}^{+}, \lim _{n \rightarrow \infty} h(n)=\infty, k>0$, and $s$ is any finite $\{M, F\}$ sequence, there is some $e>0$ such that $s \frown M^{e} F$ is impossible in $T_{h}$ at height $k$.

Proof. We may assume $s$ nonempty. We may also assume the first gender in $s$ is $M$, the other case being similar. Let $\ell=\operatorname{length}(s)$. By Lemma 17, there is some $e>1$ such that $e-1$ is not of the form $a+\sum_{p=1}^{b} h(c+p)$ for any $a, b, c \in \mathbb{N}$ with $c \leqslant \ell+k$ and $a \leqslant \max \{h(1), \ldots, h(\ell+k)\}$. Thus $e$ itself is not of the form $a+1+\sum_{p=1}^{b} h(c+p)$ for any such $a, b, c$. We will show $s \frown M^{e} F$ is impossible in $T_{h}$ at height $k$. Suppose not: suppose there is a finite directed path $v_{0}, \ldots, v_{\ell}, v_{\ell+1}, \ldots, v_{\ell+e}$ in $T_{h}$, gendered by $s \frown M^{e} F$, with $v_{0}=\widehat{m}_{k}$.

We would like to estimate in which generation does $v_{\ell}$, the first vertex corresponding to the $M^{e} F$ block, lie. We will be content with an overestimate. By Lemma 15 part 3, every edge either ends in the same generation where it began, or at most one generation further. Thus $v_{0}=\widehat{m}_{k}$ is in at most the $k$ th generation, and $v_{\ell}$ is in at most the $(k+\ell)$ th generation.

I claim $e$ has the form $a+1+\sum_{p=1}^{b} h(c+p)$ for some $a, b, c \in \mathbb{N}$ with $c \leqslant \ell+k$, $a \leqslant \max \{h(1), \ldots, h(\ell+k)\}$, a contradiction. Let $i, j$ be such that $v_{\ell}$ is in $G_{i}$ (so $i \leqslant k+\ell$ ) and $v_{\ell+e-1}$ is in $G_{j}$. Since $v_{\ell+e}$ is female, and $v_{\ell+e-1}$ is male, by Lemma 15 part 2, $v_{\ell+e-1}$ must be $m_{1}^{j}$. Thus $B=\left(v_{\ell}, \ldots, v_{\ell+e-1}\right)$ is a finite directed path of male vertices beginning with $m_{x}^{i}$ for some $x$ and ending with $m_{1}^{j}$. All of these males lie in generations between $i$ and $j$ inclusive, and for every $i<p<j$, Lemma 15 part 4 says that $B$ contains all the males in $G_{p}$. Let us count the vertices in $B$ :

1. The number of males from $G_{i}$ included in $B$ is at most all of them, so $B$ has $\leqslant h(i)$ vertices from $G_{i}$. 
2. For $i<p<j, B$ contains all $h(p)$ males of $G_{p}$. This is a total of

$$
h(i+1)+\cdots+h(i+(j-i-1))=\sum_{p=1}^{j-i-1} h(i+p)
$$

vertices.

3. $B$ has exactly one vertex from $G_{j}$, namely $m_{1}^{j}$.

Thus, the number $e$ of vertices in $B$ has the form $a+1+\sum_{p=1}^{b} h(c+p)$ for some natural $c \leqslant i \leqslant \ell+k$, some $a \leqslant \max \{h(1), \ldots, h(\ell+k)\}$, and some natural $b$, the desired contradiction.

Corollary 19. There is a biologically avoidable sequence.

Proof. Let $h$ be as in Definition 14. Using Proposition 18 repeatedly, define finite sequences $\left\{s^{k}\right\}_{k>0}$ such that each $s^{k+1}$ strictly extends $s^{k}$ and each $s^{k}$ is impossible in $T_{h}$ at height $k$. Then $s=\cup s^{k}$ is biologically avoidable by Lemma 13 .

Example 20. $M^{9} F M^{4200} F \cdots M^{e_{n}} F \cdots$ is biologically avoidable, where each $e_{n}>0$ is chosen minimal so as to avoid the form $a+1+\sum_{p=1}^{b}(c+p)(a, c \leqslant u$ where $u=\ell+n$, where $\ell$ is the length of $\left.M^{9} F \cdots M^{e_{n-1}} F\right)$.

The above example is suboptimal, because our proof of Proposition 18 used such staggering overestimates.

Example 21. An alternate way to obtain avoidable sequences is to follow the proof of Corollary 19, but to obtain $s_{k+1}$, rather than follow the instructions in Proposition 18, we can simply do a brute-force search to find the minimal $e>0$ such that $s_{k} \frown M^{e} F$ is impossible at height $k+1$ (Proposition 18 says we won't get stuck). If we do this for the function $h(n)=n$, we obtain the avoidable sequence $M^{3} F M^{5} F M^{8} F M^{11} F \cdots$ where each block of $M^{\prime}$ 's is 3 longer than the last, with one exception at the beginning (the proof is tedious so we omit it). That one exception is annoying, so here's how to further optimize the sequence: choose each $s_{k}$ to be impossible at height $k+1$. This leaves open the possibility, a priori, the sequence could occur in $T_{h}$ at height 1 . However, by fortune, it ends up being impossible at height 1 anyway. This yields a very nice well-behaved avoidable sequence,

$$
M^{2} F M^{5} F \cdots M^{3 n-1} F \cdots
$$

(again we omit the formal proof).

All the avoidable sequences we obtain in this manner have the property that they contain arbitrarily long blocks of one gender. 


\section{There is a biologically avoidable sequence in which no block of males or females has length more than 2}

Julian Ziegler Hunts discovered an interesting family of populations which witness the avoidability of sequences with very short blocks of males and females.

Definition 22. Suppose $h: \mathbb{N}^{+} \rightarrow \mathbb{N}^{+}$. The infinite 2-gendered population $H_{h}$ is defined in the same way as $T_{h}$ was defined (Definition 14) except for its edges, which are instead defined as follows.

- $m_{1}^{1}$ and $f_{1}^{1}$ have no parents.

- $\forall n>0, m_{1}^{n+1}$ has parents $m_{1}^{n}$ and $f_{h(n)}^{n}$.

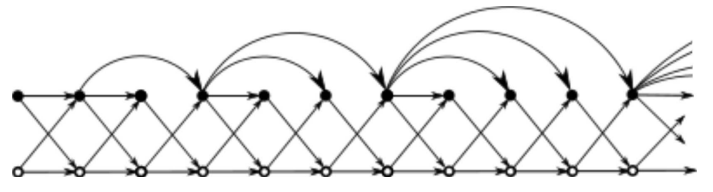

- $\forall n>0, f_{1}^{n+1}$ has parents $m_{h(n)}^{n}$ and $f_{h(n)}^{n}$

Figure 2: The infinite 2gendered population $H_{n \mapsto n}$. Solid and open vertices corre-

- $\forall n>0, \forall 0<i<h(n), m_{i+1}^{n}$ has parents $m_{1}^{n}$ and $f_{i}^{n}$. spond to males and females, not necessarily in that order.

- $\forall n>0, \forall 0<i<h(n), f_{i+1}^{n}$ has parents $f_{i}^{n}$ and $m_{i}^{n}$.

Lemma 23. Let $h: \mathbb{N}^{+} \rightarrow \mathbb{N}^{+}$.

1. $H_{h}$ is an infinite 2 -gendered population with roots $m_{1}^{1}$ and $f_{1}^{1}$.

2. If $m_{i}^{n}$ has a son, then $i=1$.

3. No edge skips an entire generation: if an edge of $H_{h}$ has initial vertex in $G_{i}$ then its terminal vertex is in $G_{i}$ or $G_{i+1}$.

4. If $B=\left(v_{1}, \ldots, v_{n}\right)$ is a finite directed-path in $H_{h}$, whose genders are alternating, and if $v_{1}$ lies in $G_{i}$ and $V_{n}$ lies in $G_{j}$, then for every $i<p<j$ and every $0<k \leqslant h(p)$, precisely one of $\left\{m_{k}^{p}, f_{k}^{p}\right\}$ appears in $B$.

Proof. Left to the reader.

We define the heights of $H_{h}$, and the corresponding $\widehat{m}_{i}$ and $\widehat{f}_{i}$, in the same way as we did for $T_{h}$.

Proposition 24. Let $h: \mathbb{N}^{+} \rightarrow \mathbb{N}^{+}$be such that $\lim _{n \rightarrow \infty} h(n)=\infty$ and $h(n)$ is even for every $n$. If $k>0$ and $s$ is any finite $\{M, F\}$-sequence, then there is some $e>0$ such that $s \frown(F M)^{e} M$ is impossible in $H_{h}$ at height $k$. 
Proof. We may assume $s$ nonempty and that the first gender in $s$ is $M$. Let $\ell=\operatorname{length}(s)$. Since $h(n)$ is even for every $n$, by Lemma 17 there is an $e>1$ such that $e-1$ is not of the form $a+\sum_{p=1}^{b} \frac{1}{2} h(c+p)$ for any $a, b, c \in \mathbb{N}$ with $c \leqslant \ell+k$ and $a \leqslant \max \{h(1) / 2, \ldots, h(\ell+$ $k) / 2\}$. Thus $e$ itself is not of the form $a+1+\sum_{p=1}^{b} \frac{1}{2} h(c+p)$ for any such $a, b, c$. We will show $s \frown(F M)^{e} M$ is impossible in $H_{h}$ at height $k$. If not, there is a finite directed path $v_{0}, \ldots, v_{\ell}, v_{\ell+1}, \ldots, v_{\ell+2 e}$ in $H_{h}$, gendered by $s \frown(F M)^{e} M$, with $v_{0}=\widehat{m}_{k}$.

By similar reasoning to the proof of Proposition 18, $v_{\ell}$ is in at most the $(k+\ell)$ th generation. I claim $e$ has the form $a+1+\sum_{p=1}^{b} \frac{1}{2} h(c+p)$, some $a, b, c$ as above, a contradiction. Let $i, j$ be such that $v_{\ell} \in G_{i}$ (so $\left.i \leqslant k+\ell\right)$ and $v_{\ell+2 e-1} \in G_{j}$. Since $v_{\ell+2 e-1}$ is male and has a son $v_{\ell+2 e}$, Lemma 23 part 2 ensures $v_{\ell+2 e-1}=m_{1}^{j}$. Thus $B=\left(v_{\ell}, \ldots, v_{\ell+2 e-1}\right)$ is a finite directed path of alternating gender beginning with $f_{x}^{i}$ for some $x$ and ending with $m_{1}^{j}$. All these vertices lie in generations between $i$ and $j$ inclusive, and for each $i<p<j$, Lemma 23 part 4 implies that the number of vertices in $B \cap G_{p}$ is exactly $h(p)$. Count the male vertices in $B$ :

1. The number of males from $G_{i}$ is at most half of them (since $B$ alternates genders), that is at most $h(i) / 2$.

2. For any $i<p<j$, the number of males from $G_{p}$ is exactly half of them, $h(p) / 2$, by Lemma 23 part 4 since $B$ alternates genders.

3. There is exactly one male from $G_{j}$, namely $m_{1}^{j}$.

Thus the number of males in $B$ is of the form $a+1+\sum_{p=1}^{b} \frac{1}{2} h(c+p)(a, b, c$ as above). But the number of males in $B$ is $e$-absurd.

Corollary 25. There is a biologically avoidable sequence in which no gender occurs thrice in a row.

Proof. Similar to the proof of Corollary 19.

\section{Application to cellular automata}

In his paper, Nathaniel Johnston [11] proved that in certain (a large family) of Conway's Life-like games, spaceships have an orthogonal speed limit of $1 / 2$ cells per generation, and a diagonal speed limit of $1 / 3$ cells per generation. We will give an alternate proof using a technique which, we believe, might be generalizable to obtain results of a wide variety ${ }^{1}$. We assume a novice-level familiarity with Life-like games (see Eppstein [7]), and brush formal details under the rug.

Definition 26. Suppose a Life-like game is played, with some initial configuration in generation 1, which generates a configuration in generation 2, and so on. A lifeline for this gameplay is a sequence $c_{1}, c_{2}, \ldots$ of cells such that:

\footnotetext{
${ }^{1}$ If nothing else, our proof would generalize with minimal changes to Life-like games in higher dimensions (see Bays [3]).
} 
1. Each $c_{i}$ is alive in generation $i$.

2. For each $i$, either $c_{i}=c_{i+1}$ or $c_{i}$ is adjacent to $c_{i+1}$.

Thus, a lifeline is a (not necessarily simple) stroll through the cells which, at each $i$ th step, visits a cell alive in the $i$ th generation.

Lemma 27. (Two Forbidden Directions) Suppose $x, y$ are any two of the following directions:

$$
N, S, E, W, N E, N W, S E \text {, and } S W \text {. }
$$

Consider a Life-like gameplay with the following properties:

1. The initial configuration is finite.

2. Each generation contains at least one live cell.

3. Birth requires $\geqslant 3$ neighbors and survival requires $\geqslant 1$ neighbor.

For any such gameplay, there is a lifeline which never steps in direction $x$ or $y$ (that is, $x_{i+1}$ is never located in the $x$ or $y$ direction from $x_{i}$ ).

To be clear, the third condition is to be understood as liberally as possible, making the lemma apply not only to the ruleset $B 345678 / S 12345678$, but to any sub-ruleset thereof.

We postpone the proof (using biologically unavoidable sequences) of Lemma 27 so we can first see how it applies to spaceship speed limits.

Theorem 28. (Johnston 2010) For any Life-like ruleset where birth requires $\geqslant 3$ neighbors and survival requires $\geqslant 1$ neighbor, spaceships can move at most $\frac{1}{2}$ cells per generation orthogonally and at most $\frac{1}{3}$ cells per generation diagonally.

Proof sketch. Since a spaceship is initially finite and does not go extinct, the hypotheses of Lemma 27 are met.

(Orthogonal) By symmetry, it's enough to show the spaceship cannot exceed $\frac{1}{2}$ cells per generation northward. By Lemma 27, there is a lifeline which never steps in direction $\mathrm{N}$ or NE. The cells in this lifeline are living cells, hence cells in the spaceship, and it follows that the spaceship cannot travel faster than the lifeline. The only movement the lifeline can make with northward component is NW (N and NE being forbidden). Any such step also moves the lifeline westward, and so to maintain an overall northward direction, any such step must be compensated for by a step in one of the directions $\mathrm{E}$ or $\mathrm{SE}$ (NE is forbidden). So at least two total steps are required per unit of overall northward movement. Thus the speed limit, $\frac{1}{2}$.

(Diagonal) By symmetry, it's enough to show the spaceship cannot exceed $\frac{1}{3}$ cells per generation northeastward. By Lemma 27, there is a lifeline which never steps $\mathrm{N}$ or $\mathrm{NE}$. The only way the lifeline may move northward is by moving NW, and two eastward steps must be added to produce overall NE movement. Thus the speed limit, $\frac{1}{3}$. 
Proof sketch of Lemma 2\%. Let $V$ be the set of pairs $(c, i)$ such that $c$ is a cell alive in generation $i$. Direct an edge from $(c, i)$ to $(d, j)$ if $j=i+1$ and either $c=d$ or $c$ is a neighbor of $d$. By (3) of Lemma 27, if $d$ is a cell born into generation $i+1$, there must have been at least three distinct neighbors $c_{1}, c_{2}, c_{3}$ of $d$ alive in generation $i$. There are only two forbidden directions, so (possibly relabelling) we may assume $d$ does not lie in a forbidden direction from $c_{1}$. Gender the edge $\left(\left(c_{1}, i\right),(d, i+1)\right)$ male, and gender all other edges terminating in $(d, i+1)$ female. If $d$ survives into generation $i+1$, then gender the edge $((d, i),(d, i+1))$ male and gender all other edges terminating in $(d, i+1)$ female. Let each vertex $(c, i)$ have birthdate $i$. The reader may check (using the hypotheses of Lemma 27) this makes $V$ an infinite 2-gendered population. By Theorem 10, there is an infinite directed path through this population with all edges male. By construction, male edges never step in a forbidden direction.

\section{$6 \quad$ Further Questions}

If $s$ is an avoidable gender sequence, to what extent can we find populations avoiding $s$ which are universal among all such, in a way analogous to that described by Cherlin and Shelah [5]?

Given a possibly avoidable sequence, can the infinite gendered populations which realize that sequence be characterized in some way? Of particular interest would be a characterization in terms of ordinal numbers, similar to R. Schmidt's characterization of rayless graphs (see Halin [9]).

But perhaps the most important question remaining is, what are the biologically unavoidable sequences? Are there any which are not eventually periodic?

\section{References}

[1] Samuel Alexander. On Guessing Whether a Sequence has a Certain Property. J. Int. Seq. 14, 12 pages, 2011. arXiv:1011.6626

[2] Samuel Alexander. Infinite graphs in systematic biology, with an application to the species problem. To appear in Acta Biotheoretica. arXiv:1201.2869

[3] Carter Bays. Candidates for the Game of Life in Three Dimensions. Comp. Syst. 1:373-400, 1987.

[4] David Cantor and Basil Gordon. Sequences of Integers with Missing Differences. J. Combinatorial Theory Ser. A, 12:281-287, 1973.

[5] Gregory Cherlin and Saharon Shelah. Universal graphs with a forbidden subtree. J. Combinatorial Theory Ser. B, 97:293-333, 2007. arXiv:math/0512218

[6] Andreas Dress, Vincent Moulton, Mike Steel, and Taoyang Wu. Species, clusters and the 'Tree of Life': A graph-theoretic perspective. J. Theoretical Biology, 265:535-542, 2010. arXiv:0908.2885 
[7] David Eppstein. Searching for Spaceships. MSRI Publications, 42:433-453, 2002. arXiv:cs/0004003

[8] Soma Gupta. Sets of Integers with Missing Differences. J. Combinatorial Theory Ser. A, 89:55-69, 2000.

[9] Rudolf Halin. The Structure of Rayless Graphs. Abh. Math. Sem. Univ. Hamburg, 68:225-253, 1998.

[10] Willi Hennig. Phylogenetic Systematics. University of Illinois press, Urbana, 1966.

[11] Nathaniel Johnston. The B36/S125 " $2 \times 2$ " Life-Like Cellular Automaton. In Andrew Adamatzky (editor), Game of Life Cellular Automata, 99-114, 2010. arXiv: 1203.1644

[12] Dina Kornet, Hans Metz, and Harold Schellinx. Internodons as equivalence classes in genealogical networks: building-blocks for a rigorous species concept. J. Math. Biology, 34:110-122, 1995.

[13] Đuro Kurepa. Ensembles ordonnés et ramifiés. Doctoral dissertation. Publ. Math. Univ. Belgrade, 4:1-138, 1935.

[14] Melvyn Nathanson. A Short Proof of Cauchy's Polygonal Number Theorem. Proc. Amer. Math. Soc., 99:22-24, 1987.

[15] Stephen Simpson. Subsystems of Second Order Arithmetic. Perspectives in Logic, Cambridge University Press, 1982.

[16] Bernd Sturmfels. Can biology lead to new theorems? Annual report of the Clay Mathematics Institute, 2005.

[17] William Wadge. Reducibility and Determinateness on the Baire Space. Doctoral dissertation, UC Berkeley, 1983. 